\title{
Tenossinovite de tornozelo em artrite reumatoide: avaliação clínica e ultrassonográfica
}

\author{
Ankle tenosynovitis in rheumatoid arthritis: clinical and ultrasonographic evaluation
}

Ana Luiza Naves Pereira ${ }^{1}$, Mariana dos Santos Pastori ${ }^{1}$, Nathalia dos Santos Vianna de Matos Leite ${ }^{1}$, Talita Rombaldi Pereira' ${ }^{1}$, Tamara Thais Kawamoto ${ }^{1}$, Karine Kefler Ferreira ${ }^{1}$, Gilberto Santos Novaes ${ }^{1}$

\begin{abstract}
RESUMO
Objetivo: Avaliar a tenossinovite de tornozelo em pacientes com artrite reumatoide com relação à presença do acometimento, ao tipo de tendão envolvido e à concordância, ou não, entre os achados clínicos e ultrassonográficos. Métodos: Vinte pacientes com artrite reumatoide e dor ou tumefação em tornozelo foram avaliados. Tendões foram analisados por meio de ultrassonografia radiográfica. A incapacidade foi avaliada pelo questionário de avaliação de saúde (HAQ). A idade, o sexo, a duração da doença e as atividades laborativas foram obtidas. Na análise estatística, utilizou-se o teste exato de Fisher. O nível de significância foi de 0,05. Resultados: Tenossinovite esteve presente em 13 de $20(65,0 \%)$ dos pacientes em 19 articulações, sendo bilateral em $6(46,1 \%)$ e unilateral em $7(53,8 \%)$. Tenossinovite do tendão tibial posterior foi observada em nove (45,0\%) pacientes, calcanear (aquileu) em 7 (35\%), tibial anterior em $3(15 \%)$ e fibular em $3(15 \%)$. Houve concordância entre o tornozelo sintomático e os achados ultrassonográficos em 92,3\% dos pacientes com tenossinovite. O HAQ não mostrou associação com o envolvimento tendíneo ( $p>0,05$ ). A duração da doença não foi associada com a tenossinovite. Os pacientes eram predominantemente idosos, com média de idade de 50,8 anos, e do sexo feminino. A duração da doença nos pacientes apresentou média de 11,4 anos e, em sua maioria, os sujeitos não realizavam atividades laborativas $(65,0 \%)$. Conclusões: Os resultados indicam que a tenossinovite do tornozelo é comum em pacientes com artrite reumatoide, seja unilateral ou bilateral, sendo o envolvimento do tendão tibial posterior o mais frequente. Finalmente, na maioria dos pacientes, verificou-se concordância entre os achados clínicos e ultrassonográficos. Palavras-chave: artrite reumatoide; tenossinovite; tornozelo; ultrassonografia.
\end{abstract}

\begin{abstract}
Objective: To investigate ankle tenosynovitis in rheumatoid arthritis patients, regarding its presence, the kind of tendon involved and the concordance between clinical and ultrasound findings. Methods: Twenty patients with rheumatoid arthritis and pain or swollen ankle joint were evaluated. Tendon involvement was evaluated with ultrasound imaging. The Health Assessment Questionnaire (HAQ) was performed for disability evaluation. Age, sex, disease duration, and vocational activity levels were also obtained. The statistical analysis included Fisher's exact test. The significance level was 0.05 . Results: Tenosynovitis was found in 13 of $20(65.0 \%)$ patients in 19 joints, in which 6 were bilaterally $(46.1 \%)$ and unilateral in $7(53.8 \%)$. Tibialis posterior tenosynovitis was seen in nine (45.0\%) patients, Achilles tenosynovitis in seven $(35.0 \%)$, tibialis anterior tenosynovitis in three $(15.0 \%)$, and peroneal tenosynovitis in three (15.0\%) patients. We found concordance between symptomatic ankle and ultrasonographic findings in $92.3 \%$ of the patients with tenosynovitis. Association between severe HAQ with tendon involvement was not found ( $\mathrm{p}>0.05)$. Disease duration was not associated with tenosynovitis. Patients were predominantly older, female, with mean age around 50.8 years. The long disease duration of patients presented a mean of 11.4 years and, most of them, with no vocational activity (65.0\%). Conclusions: The results indicate that ankle tenosynovitis is very common in rheumatoid arthritis patients, both unilateral and bilateral. Tibialis posterior was the most common tendon involvement found. Finally, we found concordance between the clinical and ultrasound findings in almost all rheumatoid arthritis patients with ankle tenosynovitis.

Keywords: arthritis, rheumatoid; tenosynovitis; ankle; ultrasonography.
\end{abstract}

'Pontifícia Universidade Católica de São Paulo (PUC-SP), Faculdade de Ciências Médicas e da Saúde - Sorocaba (SP), Brasil. Contato: gnovaes52@gmail.com

Recebido em 04/02/2016. Aceito para publicação em 26/04/2016. 


\section{INTRODUÇÃO}

A artrite reumatoide (AR) é uma doença crônica, sistêmica e autoimune, cujas alterações predominantes ocorrem nas estruturas articulares, manifestando-se por sinais flogísticos inequívocos. Embora se desenvolva predominantemente na membrana sinovial, outras estruturas articulares podem ser acometidas, tais como cápsulas fibrosas, osso subcondral, cartilagem articular, bursas, bainhas tendinosas e tendões. ${ }^{1}$

O tornozelo é uma estrutura complexa em função de seus ligamentos, tendões, sindesmose tíbio-fibular e articulação tíbio-társica atuarem em conjunto, o que proporciona os movimentos de dorsiflexão, flexão plantar, inversão e eversão apoiada nos movimentos mínimos da fíbula e da tíbia durante a marcha. ${ }^{2,3}$ Entre as manifestações extra-articulares mais comumente observadas na AR está o acometimento tendíneo das articulações, e entre essas participa o tornozelo. No entanto, ainda que presentes, são pouco frequentes os estudos, seja de avaliações clínicas ou de imagem, da tenossinovite de tornozelo na AR. ${ }^{4-6}$

$\mathrm{O}$ que tem contribuído para estes estudos mais recentes é a excelente confiabilidade do estudo das estruturas tendinosas do tornozelo pela ultrassonografia de alta resolução, observada em estudos na AR e em outras doenças reumáticas. ${ }^{7-10}$

Pouca ou quase nenhuma descrição tem sido apresentada na literatura referente à tenossinovite do tornozelo na AR em relação ao tipo de acometimento tendíneo em seus variados compartimentos, a associação do grau de incapacidade e a tendinopatia, assim como a concordância, ou não, da avaliação clínica e ultrassonográfica nesses pacientes. Dessa forma, o objetivo deste estudo foi verificar tais características da tenossinovite do tornozelo em pacientes com AR.

\section{MÉTODOS}

Foram avaliados 20 pacientes com o diagnóstico de $\mathrm{AR}$, segundo critérios do "Colégio Americano de Reumatologia" (ACR), ${ }^{11}$ e que estavam em acompanhamento clínico no Ambulatório de Reumatologia do Conjunto Hospitalar de Sorocaba (CHS). O estudo foi aprovado pelo Comitê de Ética da Faculdade de Ciências Médicas e da Saúde da Pontifícia Universidade Católica de São Paulo (PUC-SP) e o termo de consentimento livre e esclarecido foi obtido de todos os pacientes prévio à realização do estudo. Foram selecionados os pacientes com AR e dor ou tumefação de tornozelo. Realizou-se exame articular do tornozelo acometido e do tornozelo contralateral com pesquisa de dor, intensidade e localização, presença de tumefação e inspeção dinâmica da articulação (movimentos ativos e passivos).

Dados clínico-demográficos e epidemiológicos foram obtidos, tais como sexo, idade, duração da doença e atividades laborativas. Foi aplicado um questionário de avaliação da saúde (HAQ). ${ }^{12} \mathrm{O}$ exame ultrassonográfico foi realizado por radiologista, sem o conhecimento da avaliação clínica do paciente. Foi utilizado um aparelho de ultrassom modelo HD7 (Philips Medical Systems, Barueri, São Paulo). Ambos tornozelos foram examinados. Os tendões fibular longo e curto, tibial posterior, tibial anterior e calcanear (aquileu) foram avaliados.
Os tendões e suas bainhas sinoviais foram analisados quanto à espessura, aos contornos, à textura, à ecogenicidade, à presença ou não de líquido, à rotura e à calcificação.

$\mathrm{Na}$ análise estatística foram utilizados análise descritiva e teste exato de Fisher. O nível de significância foi de 0,05.

\section{RESULTADOS}

A tenossinovite de tornozelo avaliado pela ultrassonografia esteve presente em 13 dos $20(65 \%)$ pacientes reumatoides ou em 19 das 40 (47,5\%) articulações avaliadas. Em 6 (46,1\%) pacientes, o acometimento tendíneo foi bilateral, em ambos os tornozelos e unilateral em $7(53,8 \%)$ dos pacientes. Envolvimento de mais de dois tendões foi observado em $3(23,0 \%)$ pacientes. Quanto ao tipo de tendão acometido, a tenossinovite do tibial posterior foi a mais frequente, sendo detectada em $9(45,0 \%)$ pacientes. Posteriormente, foram observadas tenossinovite do calcanear (aquileu) em 7 (35\%), do tibial anterior em $3(15 \%)$ e fibular em $3(15 \%)$ pacientes (Figura 1). Houve concordância entre a avaliação clínica do tornozelo sintomático, ou seja, com dor ou tumefação articular, e os achados ultrassonográficos em 92,3\% dos pacientes com tenossinovite. Os achados ultrassonográficos mais frequentes foram: espessamento do tendão, aumento de líquido na bainha do tendão e aumento de calibre com áreas de edema peritendíneo. O HAQ classificado como grave, escore maior que dois, foi verificado em 7 pacientes e não mostrou associação com a extensão do envolvimento tendíneo ( $\mathrm{p}>0,05)$.

Em geral, os pacientes eram predominantemente idosos com média de idade de 50,8 anos (35-70 anos), 16 eram do sexo feminino e 4 do masculino. A duração da doença em média foi de 11,4 anos (1 a 23 anos). Duração de doença maior do que 10 anos ou menor do que 3 anos não foi associada com a



TTP: tenossinovite do tibial posterior $(45 \%)$; TA: tenossinovite aquileu ou calcanear (35\%); TTA: tenossinovite tibial anterior (15\%); TF: tenossinovite fibular (15\%).

Figura 1. Frequência e distribuição da tenossinovite de tornozelo em pacientes com artrite reumatoide $(n=20)$ avaliados pela clínica e ultrassonografia. 
presença de tenossinovite ( $\mathrm{p}>0,05)$. Em sua maioria, $13(65 \%)$ pacientes não realizavam atividades laborativas (Tabela 1).

\section{DISCUSSÃO}

Neste estudo, verificou-se que tenossinovite de tornozelo é um achado frequente em pacientes com AR, que pode ser tanto unilateral como bilateral sem predominância de uma das formas de acometimento. $\mathrm{O}$ tendão tibial posterior foi o mais comumente envolvido, seguido pelo calcanear (aquileu), tibial anterior e fibular. Não foi detectada associação de tenossinovite com incapacidade, medida pelo HAQ, ou duração de doença nestes pacientes. Houve, na maioria dos pacientes, concordância entre a avaliação clínica e ultrassonográfica.

Poucos estudos clínicos sobre tenossinovite de tornozelo em pacientes com AR foram realizados até o momento da realização do presente artigo. Suzuki e Okamoto ${ }^{4}$ encontraram tenossinovite de tornozelo localizado no recesso medial em 46 tornozelos, no recesso lateral em 29 e no recesso anterior em 10 de 100 tornozelos avaliados. Nesse estudo, não estava especificado quais tendões foram acometidos. Os mesmos autores notaram que pacientes com acometimento de tendão tinham menor duração da doença; portanto, a tenossinovite ocorreria nas fases iniciais da AR, fato que não foi observado no presente estudo, provavelmente em função de terem sido avaliados pacientes com doença de maior duração. Em outro estudo, ${ }^{5}$ os mesmos autores detectaram tenossinovite de tornozelo em 59,0\% de seus 17 pacientes reumatoides avaliados clínica e ultrassonograficamente, enquanto nossos resultados mostram o mesmo em $65,0 \%$ dos pacientes. Nesse estudo, encontrou-se tenossinovite do tibial posterior em $41,0 \%$, do fibular em $35,0 \%$ e do aquileu em $35,0 \%$ dos pacientes. Vale ressaltar que o estudo de Suzuki e Yshihara ${ }^{5}$ é o único disponível em que foi descrita a localização do acometimento tendíneo da tenossinovite de tornozelo em pacientes reumatoides. $\mathrm{O}$ acometimento do tibial posterior e aquileu foi próximo àqueles encontrados neste estudo, porém aqui verificou-se um menor acometimento do tendão fibular $(15,0 \%)$. No estudo citado, não foi relatada tenossinovite do tendão

Tabela 1. Características clínicas e demográficas dos pacientes com artrite reumatoide estudados.

\begin{tabular}{lc}
\hline Variáveis & Pacientes $(\mathbf{n = 2 0})$ \\
\hline Idade (anos \pm desvio padrão) & $50,8 \pm 9,1$ \\
Sexo (\%) & \\
$\quad$ Masculino & 20 \\
$\quad$ Feminino & 80 \\
\hline Duração de doença & $11,4 \pm 6,3$ \\
(anos \pm desvio padrão) & \\
Atividades laborativas (\%) & 35 \\
Sim & 65 \\
Não
\end{tabular}

Valores expressos como média \pm desvio padrão e percentual (\%). tibial anterior, que foi encontrada em $15 \%$ dos pacientes da presente análise. Estudo recente ${ }^{13}$ descreve o achado de tenossinovite de tornozelo em AR em $28 \%$ de seus pacientes, em tendões do compartimento medial, sem especificar quais tendões foram acometidos.

Um importante resultado deste estudo diz respeito à concordância entre avaliação clínica de tornozelo sintomático, ou seja, com dor ou tumefação articular, e achados ultrassonográficos, o que foi observado em $92,3 \%$ dos pacientes avaliados com tenossinovite. Esse achado mostra que a ultrassonografia para avaliação de tenossinovite de tornozelo sintomático é na, grande maioria dos casos, confiável. É claro que os resultados devem ser confirmados e novos estudos serão necessários para melhor compreensão da tenossinovite de tornozelo em AR.

Em resumo, os resultados indicam que a tenossinovite de tornozelo é frequente em pacientes com AR, seja unilateral ou bilateral, sendo o envolvimento do tendão tibial posterior o mais comum. Finalmente, na maioria dos pacientes, verificouse concordância entre os achados clínicos e ultrassonográficos.

\section{AGRADECIMENTOS}

Os autores acadêmicos receberam bolsa de ajuda de custo da Comissão de Pesquisa da PUC-SP.

\section{REFERÊNCIAS}

1. Firenstein GS. Etiology and pathogenesis of rheumatoid arthritis. In: Ruddy S, Harris ED, Sledge CB, editores. Kelley's textbook of rheumatology. $6^{\text {th }}$ ed. Philadelphia, W.B. Saunders; 2001. p. 921-67.

2. Close JR. Some applications of the functional anatomy of the ankle joint. J Bone Joint Surg Am. 1956;38(4):761-81.

3. Mendonça JA, Hernandez-Diaz C. La ultrasonografía en el estudio del tobillo y pie dolorosos. Rev Chil Reumatol. 2012;28(1):47-56.

4. Suzuki T, Okamoto A. Ultrasound examination of symptomatic ankles in shorter-duration rheumatoid arthritis patients often reveals tenosynovitis. Clin Exp Rheumatol. 2013;31(2):281-4.

5. Suzuki T, Ishihara K. Achilles paratendonitis as the initial manifestation of rheumatoid arthritis. Mod Rheumatol. 2011;21(2):219-22.

6. Suzuki T, Tohda E, Ishihara K. Power Doppler ultrasonography of symptomatic rheumatoid arthritis ankles revealed a positive association between tenosynovitis and rheumatoid factor. Mod Rheumatol. 2009;19(3):235-44.

7. Schmidt WA, Hauer RW, Banzer D, Bohl-Buhler M, Braun J, Mellerowicz $\mathrm{H}$, et al. Technique and value of artrosonography in rheumatologic diagnosis. 3: ultrasound diagnosis of the ankle joint, foot and toes. $\mathrm{Z}$ Rheumatol. 2002;61(3):279-90.

8. Bruyn GA, Moller I, Garrido J, Bong D, d'Agostino MA, Iagnocco A, et al. Reliability testing of tendon disease using two different scanning methods in patients with rheumatoid arthritis. Rheumatology. 2012;51(9):1655-61. 
9. Micu MC, Serra S, Fodor D, Crespo M, Naredo E. Interobserver reliability of ultrasound detection of tendon abnormalities at the wrist and ankle in patients with rheumatoid arthritis. Rheumatology. 2011;50:1120-4.

10. Genc H, Cakit BD, Erdem IT. Ultrasonographic evaluation of tendons and enthesal sites in rheumatoid arthritis: comparison with ankylosing spondylitis and healthy subjects. Clin Rheumatol. 2005;24:272-7.

11. Arnett FC, Edworthy SM, Bloch DA, McShane DJ, Fries JF, Cooper NS, et al. The American Rheumatism Association 1987 revised criteria for the classification of rheumatoid arthritis. Arthritis Rheum. 1988;31:315-24.

12. Ferraz MB, Oliveira LM, Araujo PM, Atra E, Tugwell P. Crosscultural reliability of the physical dimension of the health assessment questionnaire. J Rheumatol. 1990;17:813-7.

13. Alsuwaidi M, Ehrenstein B, Fleck M, Hartung W. Asymptomatic versus symptomatic ankle joints in rheumatoid arthritis: a high resolution B-mode and power Doppler ultrasound study. Arthritis Care Res. 2016;68(6):861-4. 